\title{
The Use of Sumudu Transform for Solving Certain Nonlinear Fractional Heat-Like Equations
}

\author{
Abdon Atangana ${ }^{1}$ and Adem Kılıçman ${ }^{2}$ \\ ${ }^{1}$ Institute for Groundwater Studies, Faculty of Natural and Agricultural Sciences, University of the Free State, \\ 9300 Bloemfontein, South Africa \\ ${ }^{2}$ Department of Mathematics and Institute for Mathematical Research, University Putra Malaysia (UPM), \\ 43400 Serdang, Selangor, Malaysia \\ Correspondence should be addressed to Adem Kılıçman; akilicman@putra.upm.edu.my
}

Received 4 March 2013; Accepted 16 April 2013

Academic Editor: Mustafa Bayram

Copyright (C) 2013 A. Atangana and A. Kılıçman. This is an open access article distributed under the Creative Commons Attribution License, which permits unrestricted use, distribution, and reproduction in any medium, provided the original work is properly cited.

We make use of the properties of the Sumudu transform to solve nonlinear fractional partial differential equations describing heat-like equation with variable coefficients. The method, namely, homotopy perturbation Sumudu transform method, is the combination of the Sumudu transform and the HPM using He's polynomials. This method is very powerful, and professional techniques for solving different kinds of linear and nonlinear fractional differential equations arising in different fields of science and engineering.

\section{Introduction}

In the literature one can find a wide class of methods dealing with the problem of approximate solutions to problems described by nonlinear fractional differential equations, for instance, asymptotic methods and perturbation methods [1]. The perturbation methods have some limitations; for instance, the approximate solution engages series of small parameters which causes difficulty since most nonlinear problems have no small parameters at all [1]. Even though a suitable choice of small parameters occasionally lead to ideal solution, in most cases unsuitable choices leads to serious effects in the solutions [1]. Therefore, an analytical method which does not require a small parameter in the equation modeling of the phenomenon is welcome [2-4]. To deal with the pitfall presented by these perturbation methods for solving nonlinear equations, a literature review in some new asymptotic methods for the search for the solitary solutions of nonlinear differential equations, nonlinear differentialdifference equations, and nonlinear fractional differential equations is presented in [5]. The homotopy perturbation method (HPM) was first initiated by He [6]. The HPM was also studied by many authors to present approximate and exact solution of linear and nonlinear equations arising in various scientific and technological fields [7-13]. The Adomian decomposition method (ADM) [14-19] and variational iteration method (VIM) [2-4] have also been applied to study the various physical problems. The homotopy decomposition method (HDM) was recently proposed by $[20,21]$ to solve the groundwater flow equation and the modified fractional KDV equation $[20,21]$. The homotopy decomposition method is actually the combination of the perturbation method and Adomian decomposition method. Singh et al. [22] have made used of studying the solutions of linear and nonlinear partial differential equations by using the homotopy perturbation Sumudu transform method (HPSTM). The HPSTM is a combination of Sumudu transform, HPM, and He's polynomials.

\section{Sumudu Transform}

The Sumudu transform is an integral transform similar to the Laplace transform, introduced in the early 1990s by Watugala [23] to solve differential equations and control engineering problems. 
First we will summon up the following useful definitions and theorems for this integral transform operator. Note that these theorems and definitions will be used in the rest of the paper.

\subsection{Definitions and Theorems}

Definition 1. The Sumudu transform of a function $f(t)$, defined for all real numbers $t \geq 0$, is the function $F_{s}(u)$, defined by

$$
S(f(t))=F_{s}(u)=\int_{0}^{\infty} \frac{1}{u} \exp \left[-\frac{t}{u}\right] f(t) d t
$$

Definition 2. The double Sumudu transform of a function $f(x, t)$, defined for all real numbers $(x \geq 0, t \geq 0)$, is defined by

$$
\begin{aligned}
F(u, v) & =S_{2}[f(x, t),(u, v)] \\
& =\frac{1}{v u} \iint_{0}^{\infty} \exp \left[-\left(\frac{t}{v}+\frac{x}{u}\right)\right] f(x, t) d x d t .
\end{aligned}
$$

In the same line of ideas, the double Sumudu transform of second partial derivative with respect to $x$ is of form [24]

$$
\begin{aligned}
& S_{2}\left[\frac{\partial^{2} f(x, t)}{\partial x^{2}} ;(u, v)\right] \\
& \quad=\frac{1}{u^{2}} F(u, v)-\frac{1}{u^{2}} F(0, v)-\frac{1}{u} \frac{\partial F(0, v)}{\partial x} .
\end{aligned}
$$

Similarly, the double Sumudu transform of second partial derivative with respect to $t$ is of form [24]

$$
\begin{aligned}
& S_{2}\left[\frac{\partial^{2} f(x, t)}{\partial t^{2}} ;(u, v)\right] \\
& \quad=\frac{1}{v^{2}} F(u, v)-\frac{1}{v^{2}} F(u, 0)-\frac{1}{u} \frac{\partial F(u, 0)}{\partial t} .
\end{aligned}
$$

Theorem 3. Let $G(u)$ be the Sumudu transform of $f(t)$ such that

(i) $G(1 / s) / s$ is a meromorphic function, with singularities having $\operatorname{Re}[s] \leq \gamma$ and

(ii) there exist a circular region $\Gamma$ with radius $R$ and positive constants $M$ and Kwith $|G(1 / s) / s|<M R^{-K}$; then the function $f(t)$ is given by

$$
\begin{aligned}
S^{-1}(G(s)) & =\frac{1}{2 \pi i} \int_{\gamma-i \infty}^{\gamma+i \infty} \exp [s t] G\left(\frac{1}{s}\right) \frac{d s}{s} \\
& =\sum \text { residual }\left[\exp [s t] \frac{G(1 / s)}{s}\right] .
\end{aligned}
$$

For the proof see [23].

\subsection{Properties of Sumudu Transform [25-28]}

(i) The transform of a Heaviside unit step function is a Heaviside unit step function in the transformed domain $[26,27]$.

(ii) The transform of a Heaviside unit ramp function is a Heaviside unit ramp function in the transformed domain $[26,27]$.

(iii) The transform of a monomial $t^{n}$ is the scaled mono$\operatorname{mial} S\left(t^{n}\right)=n ! u^{n}[26,27]$.

(iv) If $f(t)$ is a monotonically increasing function, so is $F(u)$, and the converse is true for decreasing functions $[26,27]$.

(v) The Sumudu transform can be defined for functions which are discontinuous at the origin. In that case the two branches of the function should be transformed separately. If $f(t)$ is $C^{n}$ continuous at the origin, so is the transformation $F(u)[26,27]$.

(vi) The limit of $f(t)$ as $t$ tends to zero is equal to the limit of $F(u)$ as $u$ tends to zero provided both limits exist $[26,27]$.

(vii) The limit of $f(t)$ as $t$ tends to infinity is equal to the limit of $F(u)$ as $u$ tends to infinity provided both limits exist $[26,27]$.

(viii) Scaling of the function by a factor $c>0$ to form the function $f(c t)$ gives a transform $F(c u)$ which is the result of scaling by the same factor $[26,27]$.

\subsection{Basic Definition of Fractional Calculus}

Definition 4. A real function $f(x), x>0$, is said to be in the space $\complement_{\mu}, \mu \in \mathbb{R}$ if there exists a real number $p>\mu$, such that $f(x)=x^{p} h(x)$, where $h(x) \in C[0, \infty)$, and it is said to be in space $C_{\mu}^{m}$ if $f^{(m)} \in C_{\mu}, m \in \mathbb{N}$.

Definition 5. The Riemann-Liouville fractional integral operator of order $\alpha \geq 0$ of a function $f \in C_{\mu}, \mu \geq-1$, is defined as

$$
\begin{gathered}
J^{\alpha} f(x)=\frac{1}{\Gamma(\alpha)} \int_{0}^{x}(x-t)^{\alpha-1} f(t) d t, \quad \alpha>0, x>0, \\
J^{0} f(x)=f(x) .
\end{gathered}
$$

Properties of the operator can be found in [30-33] one mentions only the following.

For $f \in C_{\mu}, \mu \geq-1, \alpha, \beta \geq 0$, and $\gamma>-1$

$$
\begin{gathered}
J^{\alpha} J^{\beta} f(x)=J^{\alpha+\beta} f(x), \\
J^{\alpha} J^{\beta} f(x)=J^{\beta} J^{\alpha} f(x), \\
J^{\alpha} x^{\gamma}=\frac{\Gamma(\gamma+1)}{\Gamma(\alpha+\gamma+1)} x^{\alpha+\gamma} .
\end{gathered}
$$


Lemma 6. If $m-1<\alpha \leq m, m \in \mathbb{N}$ and $f \in C_{\mu}^{m}$, and $\mu \geq-1$, then

$$
\begin{gathered}
D^{\alpha} J^{\alpha} f(x)=f(x), \\
J^{\alpha} D_{0}^{\alpha} f(x)=f(x)-\sum_{k=0}^{m-1} f^{(k)}\left(0^{+}\right) \frac{x^{k}}{k !}, \quad x>0 .
\end{gathered}
$$

Definition 7 (partial derivatives of fractional order). Assume now that $f(\mathbf{x})$ is a function of $n$ variables $x_{i} i=1, \ldots, n$ also of class $C$ on $D \in \mathbb{R}_{n}$

$$
a \partial_{\underline{\mathbf{x}}}^{\alpha} f=\left.\frac{1}{\Gamma(m-\alpha)} \int_{a}^{x_{i}}\left(x_{i}-t\right)^{m-\alpha-1} \partial_{x_{i}}^{m} f\left(x_{j}\right)\right|_{x_{j}=t} d t,
$$

where $\partial_{x_{i}}^{m}$ is the usual partial derivative of integer order $m$.

Definition 8. The Sumudu transform of the Caputo fractional derivative is defined as follows [28]:

$$
\begin{array}{r}
S\left[D_{t}^{\alpha} f(t)\right]=u^{-\alpha} S[f(t)]-\sum_{k=0}^{m-1} u^{-\alpha+k} f^{(k)}\left(0^{+}\right) \\
(m-1<\alpha \leq m) .
\end{array}
$$

\section{Solution by HPSTM}

3.1. Basic Idea of HPSTM. We illustrate the basic idea of this method, by considering a general fractional nonlinear nonhomogeneous partial differential equation with the initial condition of the form of general form

$$
D_{t}^{\alpha} U(x, t)=L(U(x, t))+N(U(x, t))+f(x, t), \quad \alpha>0
$$

subject to the initial condition

$$
\begin{gathered}
D_{0}^{k} U(x, 0)=g_{k}, \quad(k=0, \ldots, n-1), \\
D_{0}^{n} U(x, 0)=0, \quad n=[\alpha],
\end{gathered}
$$

where, $D_{t}^{\alpha}$ denotes without loss of generality the Caputo fraction derivative operator, $f$ is a known function, $N$ is the general nonlinear fractional differential operator, and $L$ represents a linear fractional differential operator.

Applying the Sumudu Transform on both sides of (11), we obtain

$$
\begin{aligned}
S\left[D_{t}^{\alpha} U(x, t]\right)= & S[L(U(x, t))] \\
& +S[N(U(x, t))]+S[f(x, t)] .
\end{aligned}
$$

Using the property of the Sumudu transform, we have

$$
\begin{aligned}
S[U(x, t)]= & u^{\alpha} S[L(U(x, t))]+u^{\alpha} S[N(U(x, t))] \\
& +u^{\alpha} S[f(x, t)]+g(x, t) .
\end{aligned}
$$

Now applying the Sumudu inverse on both sides of (24) we obtain

$$
\begin{aligned}
U & (x, t) \\
& =S^{-1}\left[u^{\alpha} S[L(U(x, t))]+u^{\alpha} S[N(U(x, t))]\right]+G(x, t),
\end{aligned}
$$

where $G(x, t)$ represents the term arising from the known function $f(x, t)$ and the initial conditions [1].

Now we apply the HPM

$$
U(x, t)=\sum_{n=0}^{\infty} p^{n} U_{n}(x, t)
$$

The nonlinear term can be decomposed into

$$
N U(x, t)=\sum_{n=0}^{\infty} p^{n} \mathscr{H}_{n}(U)
$$

using the He's polynomial $\mathscr{H}_{n}(U)[17,18]$ given as

$$
\begin{array}{r}
\mathscr{H}_{n}\left(U_{0}, \ldots, U_{n}\right)=\frac{1}{n !} \frac{\partial^{n}}{\partial p^{n}}\left[N\left(\sum_{j=0}^{\infty} p^{j} U_{j}(x, t)\right)\right], \\
n=0,1,2, \ldots
\end{array}
$$

Substituting (16) and (17)

$$
\begin{aligned}
& \sum_{n=0}^{\infty} p^{n} U_{n}(x, t) \\
& =G(x, t)+p\left[S ^ { - 1 } \left[u^{\alpha} S\left[L\left(\sum_{n=0}^{\infty} p^{n} U_{n}(x, t)\right)\right]\right.\right. \\
& \left.\left.+u^{\alpha} S\left[N\left(\sum_{n=0}^{\infty} p^{n} U_{n}(x, t)\right)\right]\right]\right]
\end{aligned}
$$

which is the coupling of the Sumudu transform and the HPM using He's polynomials [1]. Comparing the coefficients of like powers of $p$, the following approximations are obtained:

$$
\begin{aligned}
& p^{0}: U_{0}(x, t)=G(x, t), \\
& p^{1}: U_{1}(x, t)=S^{-1}\left[u^{\alpha} S\left[L\left(U_{0}(x, t)\right)+H_{0}(U)\right]\right], \\
& p^{2}: U_{2}(x, t)=S^{-1}\left[u^{\alpha} S\left[L\left(U_{1}(x, t)\right)+H_{1}(U)\right]\right], \\
& p^{3}: U_{3}(x, t)=S^{-1}\left[u^{\alpha} S\left[L\left(U_{2}(x, t)\right)+H_{2}(U)\right]\right], \\
& p^{n}: U_{n}(x, t)=S^{-1}\left[u^{\alpha} S\left[L\left(U_{n-1}(x, t)\right)+H_{n-1}(U)\right]\right] .
\end{aligned}
$$


Finally, we approximate the analytical solution $U(x, t)$ by truncated series [1]

$$
U(x, t)=\lim _{N \rightarrow \infty} \sum_{n=0}^{N} U_{n}(x, t) .
$$

The above series solutions generally converge very rapidly [1, 34-37].

\section{Application}

In this section we apply this method for solving fractional differential equation in form of (11) together with (12).

Example 9. Consider the following three-dimensional fractional heat-like equation:

$$
\begin{array}{r}
\partial_{t}^{\alpha} u(x, y, z, t)=x^{4} y^{4} z^{4}+\frac{1}{36}\left(x^{2} u_{x x}+y^{2} u_{y y}+z^{2} u_{z z}\right), \\
0<x, y, z<1, \quad 0<\alpha \leq 1
\end{array}
$$

subject to the initial condition

$$
u(x, y, z, 0)=0
$$

Following carefully the steps involved in the HDM, we arrive at the following equation:

$$
\begin{aligned}
& \sum_{n=0}^{\infty} p^{n} u_{n}(x, y, z, t) \\
& =\frac{p}{\Gamma(\alpha)} S^{-1} \\
& \times\left(u^{-\alpha} \times\left[\int_{0}^{t}(t-\tau)^{\alpha-1}\right.\right. \\
& \quad \times\left(x^{4} y^{4} z^{4}+\frac{1}{36}\right. \\
& \quad \times\left(x^{2}\left(\sum_{n=0}^{\infty} p^{n} u_{n}(x, y, z, t)\right)_{x x}\right. \\
& \quad+y^{2}\left(\sum_{n=0}^{\infty} p^{n} u_{n}(x, y, z, t)\right)_{y y} \\
& \left.\left.\left.\left.\quad+z^{2}\left(\sum_{n=0}^{\infty} p^{n} u_{n}(x, y, z, t)\right)_{z z}\right)\right) d \tau\right]\right) .
\end{aligned}
$$

Now comparing the terms of the same power of $p$ yields

$$
\begin{aligned}
& p^{0}: u_{0}(x, y, z, t) \\
& p^{1}: u_{1}(x, y, z, t)=\frac{1}{\Gamma(\alpha)} \int_{0}^{t}(t-\tau)^{\alpha-1} x^{4} y^{4} z^{4} d \tau
\end{aligned}
$$

$$
p^{n}: u_{n}(x, y, z, t)
$$

$$
\begin{aligned}
& =\frac{1}{\Gamma(\alpha)} \int_{0}^{t}(t-\tau)^{\alpha-1} \\
& \quad \times\left(\frac { 1 } { 3 6 } \left(x^{2}\left(u_{n-1}\right)_{x x}\right.\right. \\
& \left.\left.\quad+y^{2}\left(u_{n-1}\right)_{y y}+z^{2}\left(u_{n-1}\right)_{z z}\right)\right) d \tau, \\
& u_{n}(x, y, z, 0)=0, \quad n \geq 2 .
\end{aligned}
$$

Thus the following components are obtained as results of the above integrals:

$$
\begin{gathered}
u_{0}(x, y, z, t)=0, \\
u_{1}(x, y, z, t)=\frac{t^{\alpha} x^{4} y^{4} z^{4}}{\Gamma(\alpha+1)}, \\
u_{2}(x, y, z, t)=\frac{t^{2 \alpha} x^{4} y^{4} z^{4}}{\Gamma(2 \alpha+1)}, \\
u_{3}(x, y, z, t)=\frac{t^{3 \alpha} x^{4} y^{4} z^{4}}{\Gamma(3 \alpha+1)}, \\
\vdots \\
u_{n}(x, y, z, t)=\frac{t^{n \alpha} x^{4} y^{4} z^{4}}{\Gamma(n \alpha+1)} .
\end{gathered}
$$

Therefore the approximate solution of equation for the first $n$ is given as

$$
u_{N}(x, y, z, t)=\sum_{n=1}^{N} \frac{t^{n \alpha} x^{4} y^{4} z^{4}}{\Gamma(n \alpha+1)}
$$

Now when $N \rightarrow \infty$, we obtained the following solution:

$$
\begin{aligned}
u(x, y, z, t) & =\sum_{n=0}^{\infty} \frac{t^{n \alpha} x^{4} y^{4} z^{4}}{\Gamma(n \alpha+1)}-x^{4} y^{4} z^{4} \\
& =x^{4} y^{4} z^{4}\left(E_{\alpha}\left(t^{\alpha}\right)-1\right)
\end{aligned}
$$


where $E_{\alpha}\left(t^{\alpha}\right)$ is the generalized Mittag-Leffler function. Note that in the case $\alpha=1$

$$
u(x, y, z, t)=x^{4} y^{4} z^{4}(\exp (t)-1) .
$$

This is the exact solution for this case.

Example 10. We consider the three-dimensional fractional wave-like equation

$$
\begin{array}{r}
\partial_{t}^{\alpha} u(x, y, z, t) \\
=x^{2}+y^{2}+z^{2}+\frac{1}{2}\left(x^{2} u_{x x}+y^{2} u_{y y}+z^{2} u_{z z}\right), \\
0<x, y, z<1, \quad 1<\alpha \leq 2
\end{array}
$$

subject to the initial condition

$$
u(x, y, z, 0)=0, \quad u_{t}(x, y, z, 0)=x^{2}+y^{2}-z^{2} .
$$

Following carefully the steps involved in the HPSTM, we arrive at the following series solutions:

$$
\begin{gathered}
u_{0}(x, y, z, t)=\left(x^{2}+y^{2}-z^{2}\right) t, \\
u_{1}(x, y, z, t)=\frac{t^{\alpha}}{\Gamma(1+\alpha)}\left(x^{2}+y^{2}-z^{2}\right), \\
u_{2}(x, y, z, t)=\frac{t^{2 \alpha}}{\Gamma(1+2 \alpha)}\left(x^{2}+y^{2}+z^{2}\right), \\
u_{3}(x, y, z, t)=\frac{t^{3 \alpha}}{\Gamma(1+3 \alpha)}\left(x^{2}+y^{2}-z^{2}\right), \\
u_{n}(x, y, z, t)=\frac{t^{n \alpha}}{\Gamma(1+n \alpha)}\left(x^{2}+y^{2}+(-1)^{n} z^{2}\right) .
\end{gathered}
$$

Therefore the approximate solution of equation for the first $n$ is given as

$$
u_{N}(x, y, z, t)=\sum_{n=1}^{N} \frac{t^{n \alpha}}{\Gamma(n \alpha+1)}\left(x^{2}+y^{2}+(-1)^{n} z^{2}\right) .
$$

Now when $N \rightarrow \infty$, we obtained the following solution:

$$
u(x, y, z, t)=\sum_{n=1}^{\infty} \frac{t^{n \alpha}}{\Gamma(n \alpha+1)}\left(x^{2}+y^{2}+(-1)^{n} z^{2}\right) .
$$

In the case of $\alpha=2$ we obtain

$$
\begin{aligned}
u(x, y, z, t)= & \left(x^{2}+y^{2}\right) \exp (t) \\
& +z^{2} \exp (-t)-\left(x^{2}+y^{2}+z^{2}\right) .
\end{aligned}
$$

This is the exact solution for this case.
Example 11. We consider the one-dimensional fractional wave-like equation

$$
\partial_{t}^{\alpha} u(x, t)=\frac{1}{2} x^{2} u_{x x}, \quad 0<x<1,1<\alpha \leq 2, t>0
$$

with the initial conditions as

$$
u(x, 0)=x^{2}
$$

Following carefully the steps involved in the HPSTM, we arrive at the following series solutions:

$$
\begin{gathered}
u_{0}(x, t)=x^{2}, \\
u_{1}(x, t)=\frac{t^{\alpha} x^{2}}{\Gamma(\alpha+1)}, \\
u_{2}(x, t)=\frac{t^{2 \alpha} x^{2}}{\Gamma(2 \alpha+1)}, \\
u_{3}(x, t)=\frac{t^{3 \alpha} x^{2}}{\Gamma(3 \alpha+1)}, \\
\vdots \\
u_{n}(x, t)=\frac{t^{n \alpha} x^{2}}{\Gamma(n \alpha+1)} .
\end{gathered}
$$

Therefore the approximate solution of equation for the first $n$ is given as

$$
u_{N}(x, t)=\sum_{n=1}^{N} \frac{t^{n \alpha} x^{2}}{\Gamma(n \alpha+1)}
$$

Now when $N \rightarrow \infty$, we obtained the following solution:

$$
u(x, t)=\sum_{n=0}^{\infty} \frac{t^{n \alpha} x^{2}}{\Gamma(n \alpha+1)}=x^{2} E_{\alpha}\left(t^{\alpha}\right),
$$

where $E_{\alpha}\left(t^{\alpha}\right)$ is the generalized Mittag-Leffler function. Note that in the case $\alpha=1$

$$
u(x, t)=x^{2} \exp (t) .
$$

This is the exact solution for this case.

Example 12. In this example we consider the twodimensional fractional heat-like equation (Figures 1 and 2)

$$
\partial_{t}^{\alpha} u(x, t)=u_{x x}+u_{y y},
$$$$
0<x, \quad y<2 \pi, \quad t>0, \quad 0<\alpha \leq 1 .
$$ 
subject to the initial condition

$$
u(x, y, 0)=\sin (x) \sin (y)
$$

Following carefully the steps involved in the HPSTM, we arrive at the following series solutions:

$$
\begin{gathered}
u_{0}(x, y, t)=\sin (x) \sin (y), \\
u_{1}(x, y, t)=-2 \frac{t^{\alpha} \sin (x) \sin (y)}{\Gamma(\alpha+1)}, \\
u_{2}(x, y, t)=4 \frac{t^{2 \alpha} \sin (x) \sin (y)}{\Gamma(2 \alpha+1)}, \\
u_{3}(x, y, t)=-8 \frac{t^{3 \alpha} \sin (x) \sin (y)}{\Gamma(3 \alpha+1)}, \\
u_{n}(x, y, z, t)=(-2)^{n} \frac{t^{n \alpha} \sin (x) \sin (y)}{\Gamma(n \alpha+1)} .
\end{gathered}
$$

Therefore the approximate solution of equation for the first $n$ is given as

$$
u_{N}(x, y, t)=\sum_{n=1}^{N}(-2)^{n} \frac{t^{n \alpha} \sin (x) \sin (y)}{\Gamma(n \alpha+1)}
$$

Now when $N \rightarrow \infty$, we obtained the following solution:

$$
u(x, y, t)=\sum_{n=0}^{\infty} \frac{(-2)^{n} t^{n \alpha} \sin (x) \sin (y)}{\Gamma(n \alpha+1)} .
$$

Note that in the case $\alpha=1$

$$
u(x, y, z, t)=\sin (x) \sin (y) \exp (-2 t)
$$

This is the exact solution for this case.

Example 13. Consider the following time-fractional derivative in $x, y$-plane as

$$
D_{t}^{\alpha} u(x, y, t)=\frac{1}{2} \nabla^{2} u(x, y, t), \quad 1<\alpha \leq 2, x, y \in \mathbb{R}, t>0
$$

subject to the initial conditions

$$
u(x, y, 0)=\sin (x+y), \quad u_{t}(x, y, 0)=-\cos (x+y) .
$$

Applying the steps involved in HPSTM as presented in Section 3.1 to (49) we obtain

$$
\begin{aligned}
& p^{0}: u_{0}(x, y, t)=\sin (x+y)-\cos (x+y) t, \\
& p^{1}: u_{1}(x, t)=S^{-1}\left[u^{\alpha} S\left(\frac{1}{2} x^{2}\left[\left(u_{0}\right)_{x x}+\left(u_{0}\right)_{y y}\right]\right)\right] \\
& =-\sin (x+y) \frac{t^{2}}{2}+\cos (x+y) \frac{t^{3}}{3 !}, \\
& p^{2}: u_{2}(x, t)=S^{-1}\left[u^{\alpha} S\left(\frac{1}{2} x^{2}\left[\left(u_{1}\right)_{x x}+\left(u_{1}\right)_{y y}\right]\right)\right] \\
& =\sin (x+y)\left[-\frac{t^{2}}{2 !}+\frac{t^{4}}{4 !}+\frac{t^{4-\alpha}}{\Gamma(5-\alpha)}\right] \\
& +\cos (x+y)\left[-\frac{t^{3}}{3 !}+\frac{t^{5}}{5 !}+\frac{t^{5-\alpha}}{\Gamma(6-\alpha)}\right] \text {, } \\
& p^{3}: u_{3}(x, t) \\
& =S^{-1}\left[u^{\alpha} S\left(\frac{1}{2} x^{2}\left[\left(u_{2}\right)_{x x}+\left(u_{2}\right)_{y y}\right]\right)\right] \\
& =\sin (x+y)\left[-\frac{t^{2}}{2 !}+\frac{t^{4}}{4 !}+\frac{t^{6}}{6 !}+\frac{2 t^{4-\alpha}}{\Gamma(5-\alpha)}-\frac{2 t^{6-\alpha}}{\Gamma(7-\alpha)}\right. \\
& \left.-\frac{4^{\alpha-2} \sqrt{\pi} t^{6-2 \alpha}}{(6-2 \alpha)(5-2 \alpha) \Gamma(3-\alpha) \Gamma(2.5-\alpha)}\right] \\
& +\cos (x+y) \\
& \times\left[\frac{t^{3}}{3 !}-\frac{t^{5}}{5 !}+\frac{t^{7}}{7 !}+\frac{2 t^{7-\alpha}}{\Gamma(7-\alpha)}+\frac{2 t^{7-2 \alpha}}{\Gamma(8-2 \alpha)}\right] .
\end{aligned}
$$

Therefore the series solution is given as

$$
\begin{aligned}
& u(x, y, t) \\
& =\sin (x+y) \\
& \times\left[1-\frac{3 t^{2}}{2 !}+\frac{t^{4}}{8}+\frac{t^{6}}{6 !}+\frac{3 t^{4-\alpha}}{\Gamma(5-\alpha)}-\frac{2 t^{6-\alpha}}{\Gamma(7-\alpha)}\right. \\
& \left.\quad-\frac{4^{\alpha-2} \sqrt{\pi} t^{6-2 \alpha}}{(6-2 \alpha)(5-2 \alpha) \Gamma(3-\alpha) \Gamma(2.5-\alpha)}\right] \\
& +\cos (x+y) \\
& \times\left[-t+\frac{t^{3}}{3 !}-\frac{t^{5}}{5 !}+\frac{t^{7}}{7 !}+\frac{3 t^{7-\alpha}}{\Gamma(7-\alpha)}+\frac{t^{7-2 \alpha}}{\Gamma(8-2 \alpha)}\right]+\cdots
\end{aligned}
$$


It is important to point out that, if $\alpha=2$, the above solution takes the form

$$
\begin{aligned}
u_{N=4}(x, y, t)= & \sin (x+y)\left[1-\frac{t^{2}}{2 !}+\frac{t^{4}}{4 !}-\frac{t^{6}}{6 !}\right] \\
& -\cos (x+y)\left[t-\frac{t^{3}}{3 !}+\frac{t^{5}}{5 !}-\frac{t^{7}}{7 !}\right]
\end{aligned}
$$

which is the first four terms of the series expansion of the exact solution $u(x, y, t)=\sin (x+y-t)$.

Example 14. Consider the following two-dimensional heatlike equation:

$$
\begin{array}{r}
D_{t t}^{\alpha} u=\frac{1}{12}\left(x^{2} u_{x x}+y^{2} u_{y y}\right), \\
0<x, \quad y<1, \quad 1<\alpha \leq 2, \quad t>0
\end{array}
$$

subject to the initial conditions

$$
u(x, y, 0)=x^{2}, \quad u_{t}(x, y, 0)=y^{2}
$$

The exact solution is given as

$$
u(x, y, t)=x^{2} \cosh (t)+y^{2} \sinh (t)
$$

Applying the Sumudu transform on both sides of (53), we obtain the following:

$$
S[u(x, y, t)]=x^{2} t^{2}+y^{2}+u^{\alpha}\left[\frac{1}{12} S\left(x^{2} u_{x x}+y^{2} u_{y y}\right)\right]
$$

Applying the inverse Sumudu transform on both sides of (56), we obtain the following:

$$
\begin{aligned}
u(x, y, t)= & x^{2} t^{2}+y^{2}+S^{-1} \\
& \times\left[u^{\alpha}\left[\frac{1}{12} S\left(x^{2} u_{x x}+y^{2} u_{y y}\right)\right]\right]
\end{aligned}
$$

Now applying the homotopy perturbation technique on the above equation we obtain the following:

$$
\begin{aligned}
& \sum_{n=0}^{\infty} p^{n} u_{n}(x, y, t) \\
& =x^{2} t^{2}+y^{2}+S^{-1}
\end{aligned}
$$

$$
\begin{array}{r}
\times\left[u ^ { \alpha } \left[\frac { 1 } { 1 2 } S \left(x^{2}\left[\sum_{n=0}^{\infty} p^{n} u_{n}(x, y, t)\right]_{x x}\right.\right.\right. \\
\left.\left.\left.+y^{2}\left[\sum_{n=0}^{\infty} p^{n} u_{n}(x, y, t)\right]_{y y}\right)\right]\right] .
\end{array}
$$

By comparing the coefficients of like powers of $p$, we have

$$
\begin{aligned}
p^{0} & : u_{0}(x, y, t)=x^{2} t^{2}+y^{2}, \\
p^{1} & : u_{1}(x, y, t) \\
& =S^{-1}\left[u^{\alpha}\left[\frac{1}{12} S\left(x^{2}\left(u_{0}\right)_{x x}+y^{2}\left(u_{0}\right)_{y y}\right)\right]\right] \\
& =x^{2} t^{2}+y^{2}+\frac{1}{6} y^{4} t^{3}+\frac{1}{2} x^{4} t^{2},
\end{aligned}
$$

$$
\begin{aligned}
p^{2}: & u_{2}(x, y, t) \\
= & S^{-1}\left[u^{\alpha}\left[\frac{1}{12} S\left(x^{2}\left(u_{1}\right)_{x x}+y^{2}\left(u_{1}\right)_{y y}\right)\right]\right] \\
= & \frac{1}{3} y^{4} t^{3}+\frac{1}{2} x^{4} t^{2}+\frac{(y t)^{4}}{24}+\frac{(y t)^{5}}{120} \\
& +\frac{x^{4} t^{5-\alpha}}{\Gamma(3-\alpha)(4-\alpha)}+\frac{y^{4} t^{5-\alpha}}{\Gamma(5-\alpha)(5-\alpha)} \\
& -\frac{x^{4} t^{4-\alpha}}{\Gamma(3-\alpha)(3-\alpha)}-\frac{y^{4} t^{5-\alpha}}{\Gamma(4-\alpha)(4-\alpha)}
\end{aligned}
$$

Example 15. Consider the following one-dimensional fractional heat-like equation:

$$
\begin{array}{r}
D_{t t}^{\alpha} u=x^{2} \frac{\partial\left[u_{x} u_{x x}\right]}{\partial x}-x^{2}\left(u_{x x}\right)^{2}-u, \\
0 \leq x \leq 1, \quad 0 \leq t, \quad 1<\alpha \leq 2
\end{array}
$$

Subject to the initial conditions

$$
u(x, 0)=0, \quad u_{t}(x, 0)=x^{2}
$$




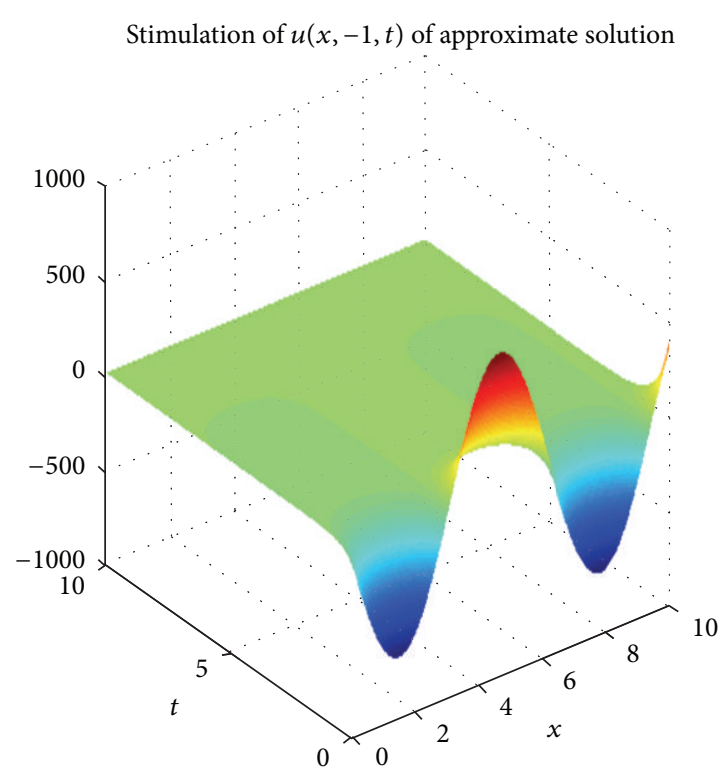

(a)

Stimulation of $u(x,-8, t)$ of approximate solution

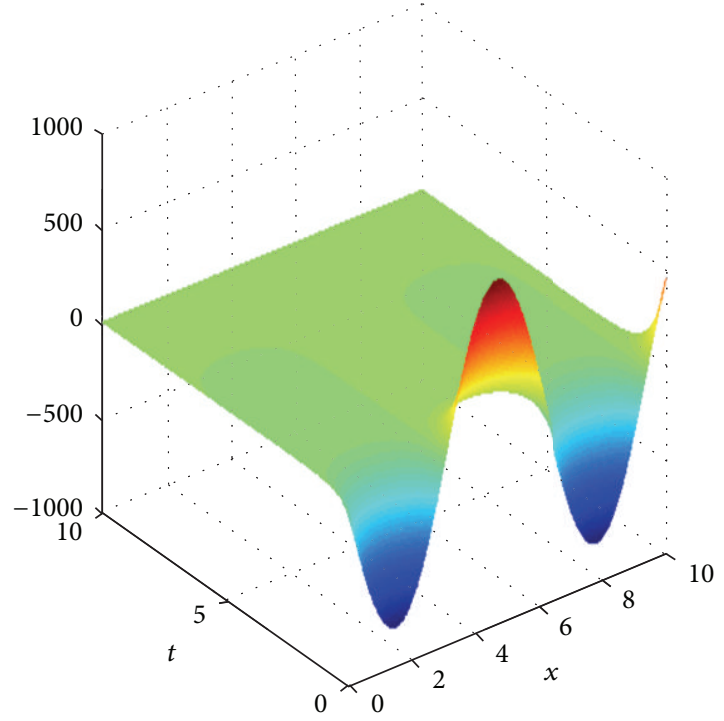

(c)

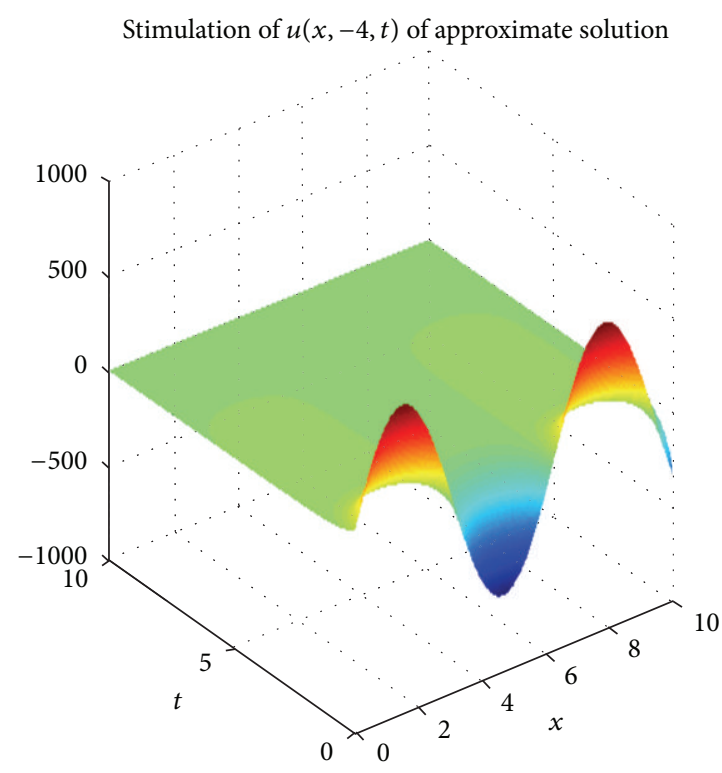

(b)

Stimulation of $u(x,-12, t)$ of approximate solution

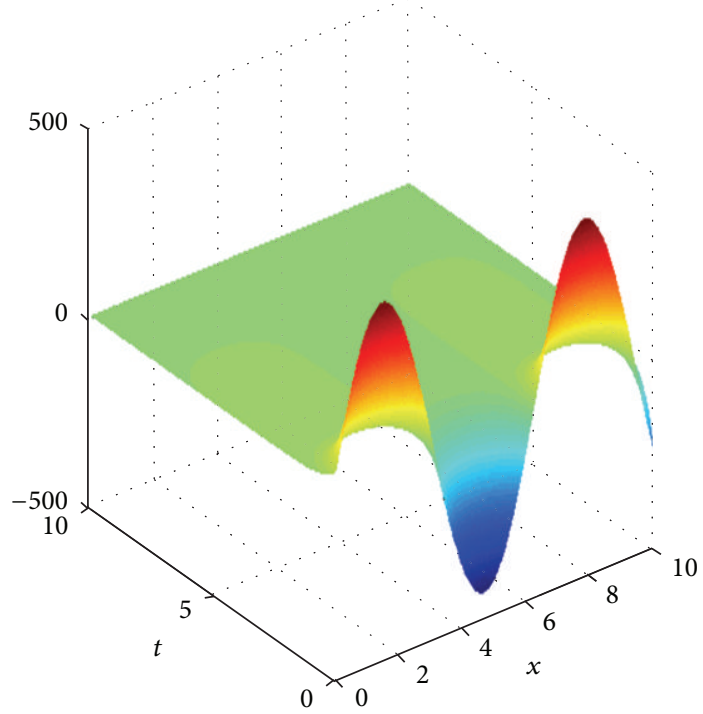

(d)

FIgURE 1: Numerical simulations of the approximate solution of (42) for a fixed $y$.

The exact solution is given as

$$
u(x, t)=x^{2} \sin [t]
$$

Applying the Sumudu transform on both sides of (60), we obtain the following:

$$
S[u(x, t)]=x^{2}+u^{\alpha}\left[x^{2} \frac{\partial\left[u_{x} u_{x x}\right]}{\partial x}-x^{2}\left(u_{x x}\right)^{2}-u\right] .
$$

Applying the inverse Sumudu transform on both sides of (63), we obtain the following:

$$
\begin{aligned}
u(x, y, t)= & x^{2}+S^{-1} \\
& \times\left[u^{\alpha}\left[S\left(x^{2} \frac{\partial\left[u_{x} u_{x x}\right]}{\partial x}-x^{2}\left(u_{x x}\right)^{2}-u\right)\right]\right] .
\end{aligned}
$$




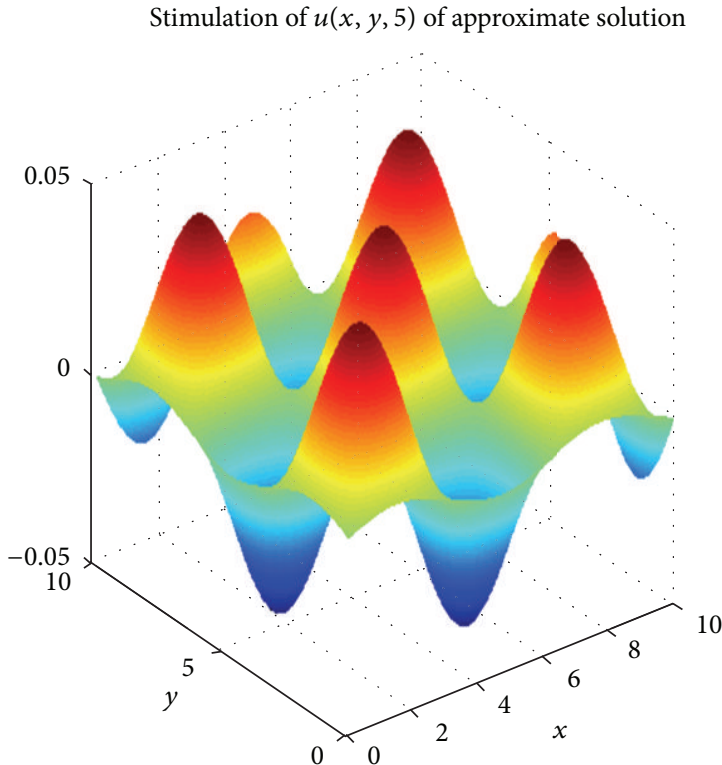

(a)

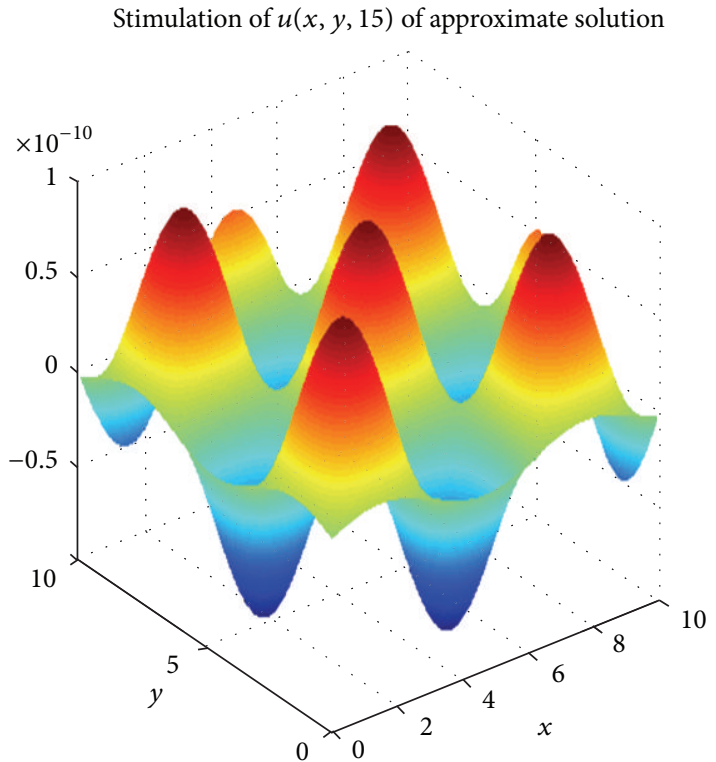

(c)
Stimulation of $u(x, y, 10)$ of approximate solution

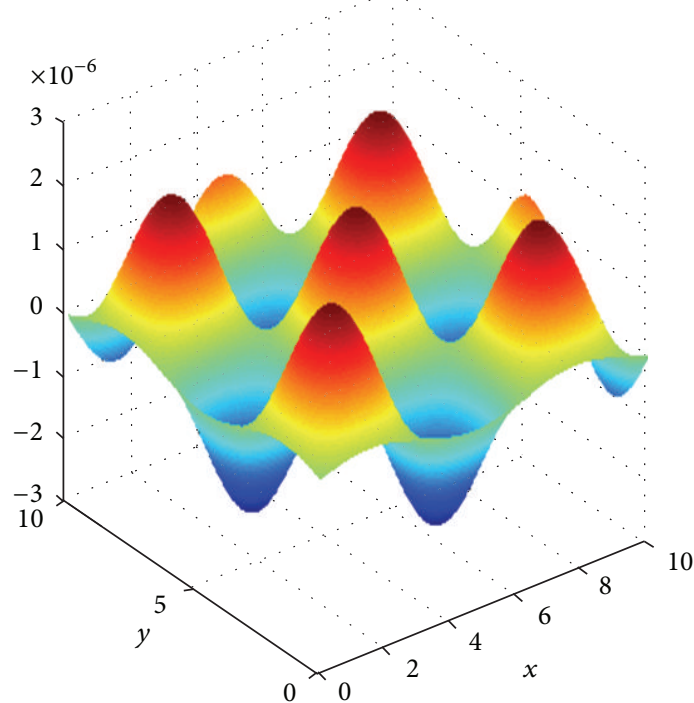

(b)

Stimulation of $u(x, y, 20)$ of approximate solution

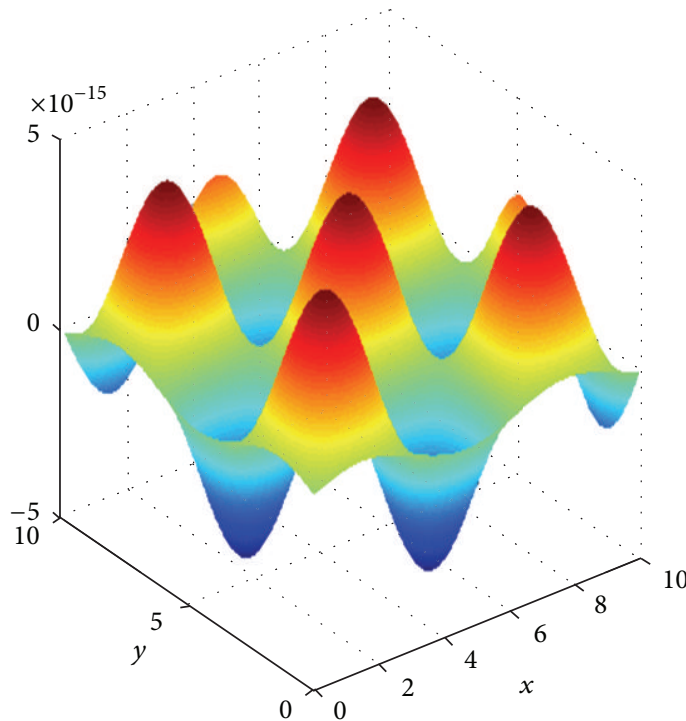

(d)

FIgURE 2: Numerical simulations of the approximate solution of (42) for a fixed $t$.

Now applying the homotopy perturbation technique on the above equation we obtain the following:

$$
\begin{gathered}
\sum_{n=0}^{\infty} p^{n} u_{n}(x, y, t) \\
=x^{2}+S^{-1}
\end{gathered}
$$

$$
\begin{gathered}
\times\left[u ^ { \alpha } \left[S \left(x^{2} \sum_{n=0}^{\infty} p^{n} H_{n}-x^{2} \sum_{n=0}^{\infty} p^{n} H_{n}^{1}\right.\right.\right. \\
\left.\left.\left.-\sum_{n=0}^{\infty} p^{n} u_{n}(x, t)\right)\right]\right] .
\end{gathered}
$$


TABLE 1: Numerical values for approximate solutions of (49) via ADM, VIM, and HPSTM.

\begin{tabular}{|c|c|c|c|c|c|c|}
\hline$t$ & $x$ & $y$ & ADM [29] & VIM [29] & HPSTM & Exact \\
\hline \multirow{4}{*}{0.25} & 0.5 & 0.5 & 0.68163209 & 0.68163219 & 0.681639 & 0.681639 \\
\hline & 0.5 & 1.0 & 0.94898215 & 0.94898245 & 0.948985 & 0.948985 \\
\hline & 1.0 & 0.5 & 0.94898215 & 0.94898245 & 0.948985 & 0.948985 \\
\hline & 1.0 & 1.0 & 0.98398623 & 0.98398643 & 0.983986 & 0.983986 \\
\hline \multirow{4}{*}{0.5} & 0.5 & 0.5 & 0.47942925 & 0.47942985 & 0.479425 & 0.479426 \\
\hline & 0.5 & 1.0 & 0.84147331 & 0.84147361 & 0.841471 & 0.841471 \\
\hline & 1.0 & 0.5 & 0.84147331 & 0.84147361 & 0.841471 & 0.841471 \\
\hline & 1.0 & 1.0 & 0.99749205 & 0.99749235 & 0.997495 & 0.997495 \\
\hline \multirow{4}{*}{0.75} & 0.5 & 0.5 & 0.2474231 & 0.2474232 & 0.247402 & 0.247404 \\
\hline & 1.0 & 1.0 & 0.68163452 & 0.68163456 & 0.681636 & 0.681639 \\
\hline & 0.5 & 0.5 & 0.68163453 & 0.68163456 & 0.681636 & 0.681639 \\
\hline & 1.0 & 1.0 & 0.94898533 & 0.94898532 & 0.948982 & 0.948985 \\
\hline \multirow{4}{*}{1.0} & 0.5 & 0.5 & -0.000001905 & -0.000001925 & -0.0000019 & -0.00000018 \\
\hline & 1.0 & 1.0 & 0.4794205 & 0.4794215 & 0.479401 & 0.479426 \\
\hline & 0.5 & 0.5 & 0.4794205 & 0.4794215 & 0.479401 & 0.479426 \\
\hline & 1.0 & 1.0 & 0.8414352 & 0.8414582 & 0.841448 & 0.841448 \\
\hline
\end{tabular}

By comparing the coefficients of like powers of $p$, we have

$$
\begin{aligned}
p^{0} & : u_{0}(x, y, t)=x^{2} \\
p^{1}: & u_{1}(x, y, t) \\
& =S^{-1}\left[u^{\alpha}\left[S\left[x^{2} H_{0}-x^{2} H_{0}^{1}-u_{0}\right]\right]\right]=-x^{2} t \\
p^{2}: & u_{2}(x, y, t) \\
= & S^{-1}\left[u^{\alpha}\left[u^{\alpha}\left[S\left[x^{2} H_{1}-x^{2} H_{1}^{1}-u_{1}\right]\right]\right]\right] \\
= & x^{2}\left[-\frac{t^{3}}{3 !}+\frac{t^{5}}{5 !}+\frac{t^{5-\alpha}}{\Gamma(6-\alpha)}\right] \\
p^{3} & : u_{3}(x, y, t) \\
& =S^{-1}\left[u^{\alpha}\left[\left[S\left[x^{2} H_{2}-x^{2} H_{2}^{1}-u_{2}\right]\right]\right]\right] \\
& \left.+\frac{3 t^{7-\alpha}}{\Gamma(7-\alpha)}+\frac{t^{7-2 \alpha}}{\Gamma(8-2 \alpha)}+\cdots+\cdots\right] \\
& =x^{2}\left[\frac{t^{3}}{3 !}-\frac{t^{5}}{5 !}+\frac{t^{7}}{7 !}+\frac{t^{3}}{\Gamma(7-\alpha)}-\frac{t^{5}}{5 !}+\frac{t^{7}}{7 !}+\frac{2 t^{7-2 \alpha}}{\Gamma(8-2 \alpha)}\right] \\
& x, t)
\end{aligned}
$$

Now if we replace $\alpha=2$, we recover the following series approximation:

$$
u(x, t)=x^{2}\left[t-\frac{t^{3}}{3 !}+\frac{t^{5}}{5 !}-\frac{t^{7}}{7 !}+\cdots+\cdots\right]=x^{2} \sin [t]
$$

which is the exact solution of this case.

\section{Conclusion}

The aim of this work was to make use of the properties of the so-called Sumudu transform to solve nonlinear fractional heat-like equations. The basic idea of the method combines Sumudu transform and the HPM using He's polynomials. In addition the method is friendly user, and it does not require anything like Adomian polynomial. From the numerical comparison in Table 1, we can see that, these three methods are very powerful, and efficient techniques for solving different kinds of linear and nonlinear fractional differential equations arising in different fields of science and engineering. However, the HPSTM has an advantage over the ADM and VIM which is that it solves the nonlinear problems without anything like the Lagrangian multiplier as in the case of VIM. We do not need to calculate anything like Adomian polynomial as in the case of ADM. In addition the calculations involved in HPSTM are very simple and straightforward.

\section{Acknowledgment}

The authors gratefully acknowledge that this research was partially supported by University Putra Malaysia under the ERGS Grant Scheme having project number 5527068. 


\section{References}

[1] J. Singh, D. Kumar, and A. Kılıçman, "Homotopy perturbation method for fractional gas dynamics equation using sumudu transform," Abstract and Applied Analysis, vol. 2013, Article ID 934060, 8 pages, 2013.

[2] G. C. Wu, "New trends in the variational iteration method," Communications in Fractional Calculus, vol. 2, pp. 59-75, 2011.

[3] G. C. Wu and D. Baleanu, "Variational iteration method for the Burgers' flow with fractional derivatives-new Lagrange multipliers," Applied Mathematical Modelling, vol. 5, pp. 10121018, 2012.

[4] G. C. Wu and D. Baleanu, "Variational iteration method for fractional calculus-a universal approach by Laplace transform," Advances in Difference Equations, vol. 2013, article 18, 2013.

[5] J. H. He, "Asymptotic methods for solitary solutions and compactons," Abstract and Applied Analysis, vol. 2012, Article ID 916793, 130 pages, 2012.

[6] J. H. He, "Homotopy perturbation technique," Computer Methods in Applied Mechanics and Engineering, vol. 178, no. 3-4, pp. 257-262, 1999.

[7] D. D. Ganji, “The application of He's homotopy perturbation method to nonlinear equations arising in heat transfer," Physics Letters A, vol. 355, no. 4-5, pp. 337-341, 2006.

[8] A. Yildirım, "An algorithm for solving the fractional nonlinear Schrödinger equation by means of the homotopy perturbation method," International Journal of Nonlinear Sciences and Numerical Simulation, vol. 10, no. 4, pp. 445-450, 2009.

[9] D. D. Ganji and M. Rafei, "Solitary wave solutions for a generalized Hirota-Satsuma coupled KdV equation by homotopy perturbation method," Physics Letters A, vol. 356, no. 2, pp. 131137, 2006.

[10] M. M. Rashidi, D. D. Ganji, and S. Dinarvand, "Explicit analytical solutions of the generalized Burger and Burger-Fisher equations by homotopy perturbation method," Numerical Methods for Partial Differential Equations, vol. 25, no. 2, pp. 409-417, 2009.

[11] J. Hristov, "A short-distance integral-balance solution to a strong subdiffusion equation: a weak power-law profile," International Review of Chemical Engineering-Rapid Communications, vol. 2, no. 5, pp. 555-563, 2010.

[12] J. Wang, Y. Khan, L. X. Lu, and Z. W. Wang, "Inner resonance of a coupled hyperbolic tangent nonlinear oscillator arising in a packaging system," Applied Mathematics and Computation, vol. 218, no. 15, pp. 7876-7879, 2012.

[13] J. Wang, Y. Khan, R. H. Yang, L. X. Lu, Z. W. Wang, and N. Faraz, "A mathematical modelling of inner-resonance of tangent nonlinear cushioning packaging system with critical components," Mathematical and Computer Modelling, vol. 54, pp. 2573-2576, 2011.

[14] A. Abdon, "New class of boundary value problems," Information Sciences Letters, vol. 1, no. 2, pp. 67-76, 2012.

[15] V. Daftardar-Gejji and H. Jafari, "Adomian decomposition: a tool for solving a system of fractional differential equations," Journal of Mathematical Analysis and Applications, vol. 301, no. 2, pp. 508-518, 2005.

[16] J. S. Duan, R. Rach, D. Bulean, and A. M. Wazwaz, "A review of the Adomian decomposition method and its applications to fractional differential equations," Communications in Fractional Calculus, vol. 3, pp. 73-99, 2012.
[17] D. Q. Zeng and Y. M. Qin, "The Laplace-Adomian-Pade technique for the seepage flows with the Riemann-Liouville derivatives," Communications in Fractional Calculus, vol. 3, pp. 26-29, 2012.

[18] G. C. Wu, "Adomian decomposition method for non-smooth initial value problems," Mathematical and Computer Modelling, vol. 54, no. 9-10, pp. 2104-2108, 2011.

[19] G. C. Wu, Y. G. Shi, and K. T. Wu, "Adomian decomposition method and non-analytical solutions of fractional differential equations," Romanian Journal of Physics, vol. 56, no. 7-8, pp. 873-880, 2011.

[20] A. Atangana and A. Secer, "Time-fractional coupled-the Korteweg-de Vries equations," Abstract and Applied Analysis, vol. 2013, Article ID 947986, 8 pages, 2013.

[21] A. Atangana and J. F. Botha, "Analytical solution of groundwater flow equation via homotopy decomposition method," Journal of Earth Science \& Climatic Change, vol. 3, article 115, 2012.

[22] J. Singh, D. Kumar, and Sushila, "Homotopy perturbation Sumudu transform method for nonlinear equations," Advances in Applied Mathematics and Mechanics, vol. 4, pp. 165-175, 2011.

[23] G. K. Watugala, "Sumudu transform: a new integral transform to solve differential equations and control engineering problems," International Journal of Mathematical Education in Science and Technology, vol. 24, no. 1, pp. 35-43, 1993.

[24] H. Eltayeb and A. K1lıçman, "A note on the Sumudu transforms and differential equations," Applied Mathematical Sciences, vol. 4, no. 22, pp. 1089-1098, 2010.

[25] S. Weerakoon, "Application of Sumudu transform to partial differential equations," International Journal of Mathematical Education in Science and Technology, vol. 25, no. 2, pp. 277-283, 1994.

[26] M. A. Asiru, "Classroom note: application of the Sumudu transform to discrete dynamic systems," International Journal of Mathematical Education in Science and Technology, vol. 34, no. 6, pp. 944-949, 2003.

[27] M. A. Aşiru, "Further properties of the Sumudu transform and its applications," International Journal of Mathematical Education in Science and Technology, vol. 33, no. 3, pp. 441-449, 2002.

[28] A. Atangana and E. Alabaraoye, "Solving system of fractional partial differential equations arisen in the model of HIV infection of CD4+ cells and attractor one-dimensional KellerSegel equation," Advances in Difference Equations, vol. 2013, article 94, 2013.

[29] D. H. Shou and J. H. He, "Beyond Adomian methods: the variational iteration method for solving heat-like and wave-like equations with variable coefficients," Physics Letters A, vol. 73, no. 1, pp. 1-5, 2007.

[30] I. Podlubny, Fractional Differential Equations, Academic Press, New York, NY, USA, 1999.

[31] A. A. Kilbas, H. M. Srivastava, and J. J. Trujillo, Theory and Applications of Fractional Differential Equations, Elsevier, Amsterdam, The Netherlands, 2006.

[32] G. Jumarie, "Table of some basic fractional calculus formulae derived from a modified Riemann-Liouville derivative for nondifferentiable functions," Applied Mathematics Letters, vol. 22, no. 3, pp. 378-385, 2009.

[33] G. Jumarie, "Laplace's transform of fractional order via the Mittag-Leffler function and modified Riemann-Liouville derivative," Applied Mathematics Letters, vol. 22, no. 11, pp. 16591664, 2009. 
[34] J. M. Tchuenche and N. S. Mbare, "An application of the double Sumudu transform," Applied Mathematical Sciences, vol. 1, no. 1-4, pp. 31-39, 2007.

[35] A. Kılıçman, H. Eltayeb, and R. P. Agarwal, "On Sumudu transform and system of differential equations," Abstract and Applied Analysis, vol. 2010, Article ID 598702, 11 pages, 2010.

[36] V. G. Gupta and B. Sharma, "Application of Sumudu transform in reaction-diffusion systems and nonlinear waves," Applied Mathematical Sciences, vol. 4, no. 9-12, pp. 435-446, 2010.

[37] F. B. M. Belgacem, A. A. Karaballi, and S. L. Kalla, "Analytical investigations of the Sumudu transform and applications to integral production equations," Mathematical Problems in Engineering, vol. 2003, no. 3, pp. 103-118, 2003. 


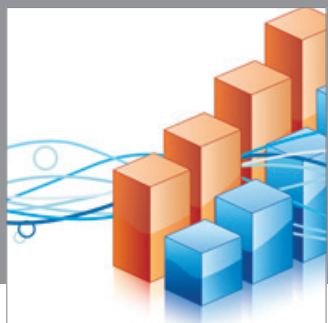

Advances in

Operations Research

mansans

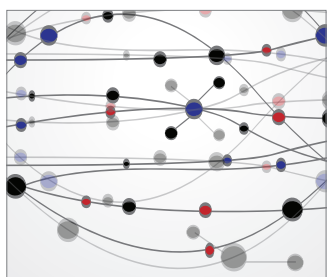

The Scientific World Journal
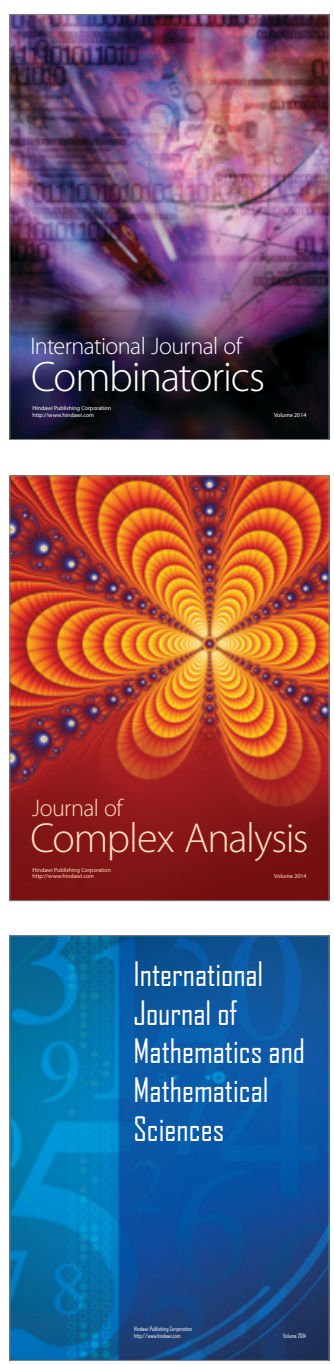
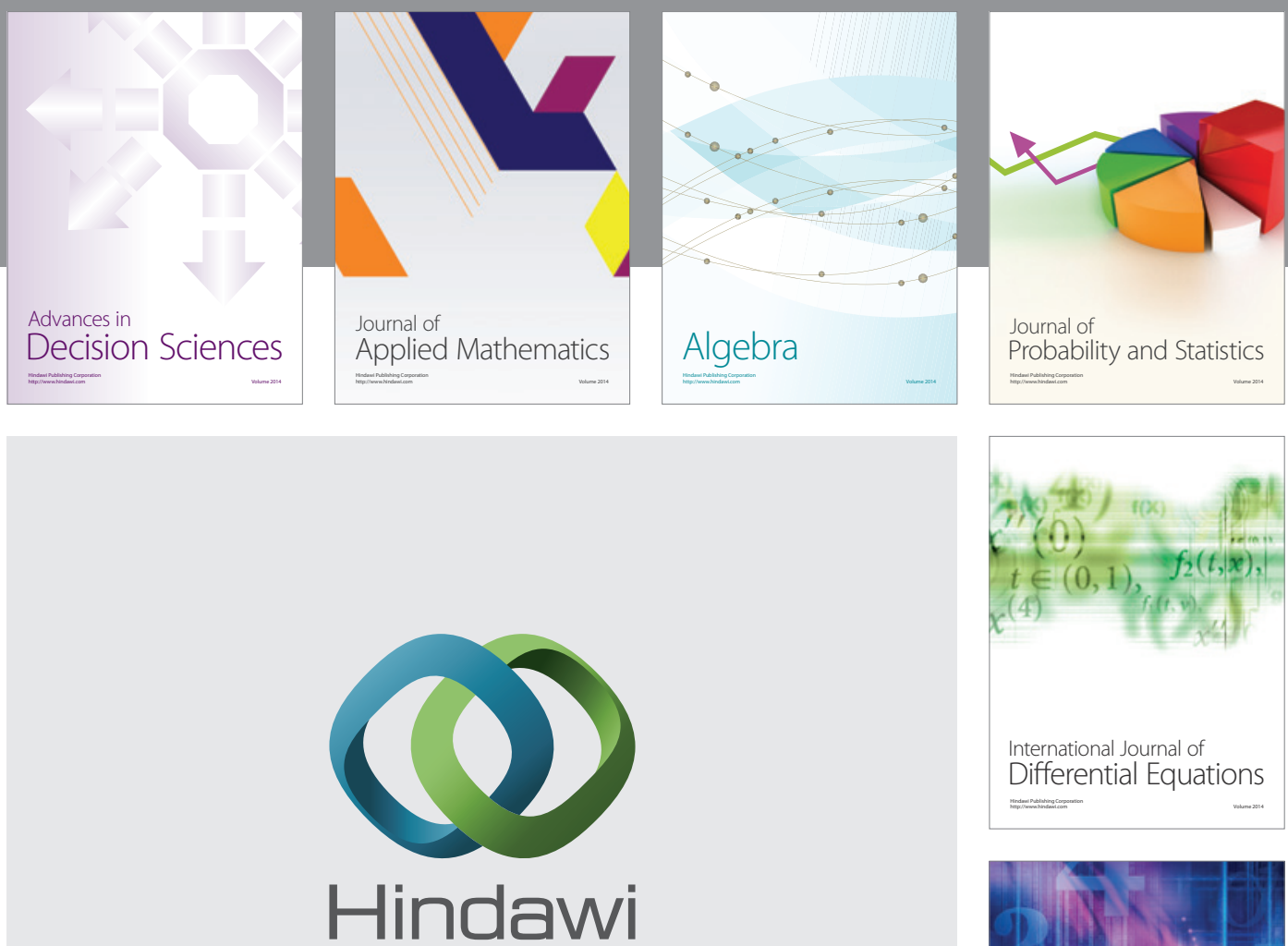

Submit your manuscripts at http://www.hindawi.com
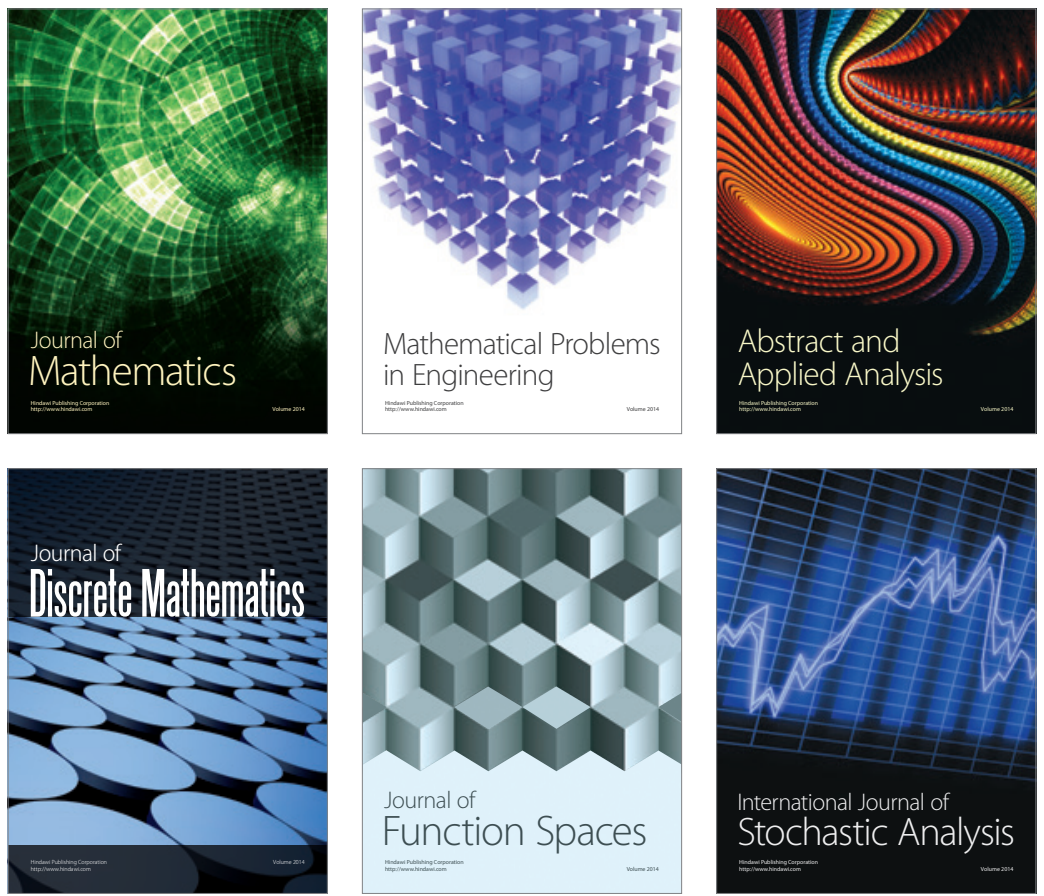

Journal of

Function Spaces

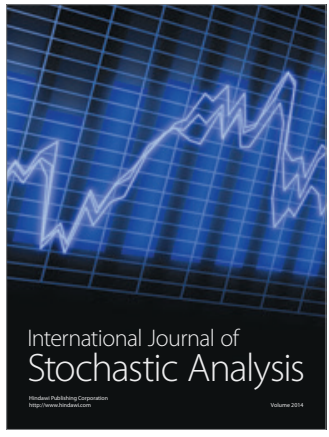

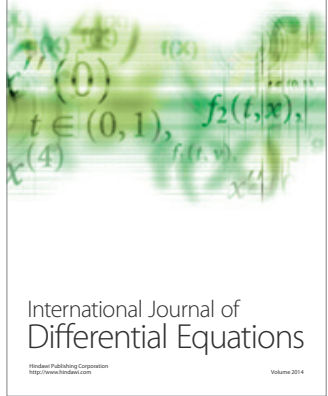
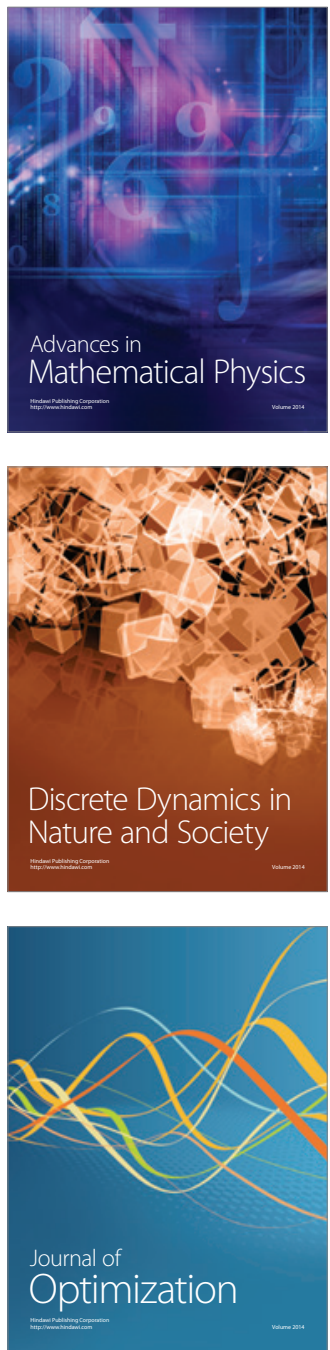